1997-10-01

\title{
System for electron paramagnetic resonance measurements at high pressure and low temperature
}

\author{
Ke Huang \\ kirkkhuang@gmail.com \\ Daniel L. Decker \\ dldecker@broadweave.net \\ H. Mark Nelson \\ J. Dean Barnett \\ jdeanbarnett@gmail.com
}

Follow this and additional works at: https://scholarsarchive.byu.edu/facpub

Part of the Astrophysics and Astronomy Commons, and the Physics Commons

\section{Original Publication Citation}

Huang, Ke, D. L. Decker, H. M. Nelson, and J. D. Barnett."System for electron paramagnetic resonance measurements at high pressure and low temperature." Review of Scientific Instruments 68 (1997): 3877-3882.

\section{BYU ScholarsArchive Citation}

Huang, Ke; Decker, Daniel L.; Nelson, H. Mark; and Barnett, J. Dean, "System for electron paramagnetic resonance measurements at high pressure and low temperature" (1997). Faculty Publications. 659. https://scholarsarchive.byu.edu/facpub/659

This Peer-Reviewed Article is brought to you for free and open access by BYU ScholarsArchive. It has been accepted for inclusion in Faculty Publications by an authorized administrator of BYU ScholarsArchive. For more information, please contact ellen_amatangelo@byu.edu. 


\title{
System for electron paramagnetic resonance measurements at high pressure and low temperature
}

\author{
Ke Huang, ${ }^{\text {a) }}$ D. L. Decker, H. M. Nelson, and J. D. Barnett \\ Department of Physics and Astronomy, Brigham Young University, Provo, Utah 84602
}

(Received 19 May 1997; accepted for publication 7 July 1997)

\begin{abstract}
Major improvements have been made on the sensitive high pressure electron paramagnetic resonance (EPR) system developed previously in 1978 at this laboratory. These changes allow low temperature measurements and provide a more stable signal. The high pressure EPR cell is placed inside a vacuum chamber to provide thermal isolation, so that the system may be cooled by a closed cycle refrigerator to temperatures between 45 and $60 \mathrm{~K}$, depending on the energy input to the modulation coil. The combination of high pressure and low temperature greatly expands the thermodynamic range over which EPR measurements can be made. An improved and effective method of forming a conductive surface to define the microwave cavity is presented. This method successfully avoids the deterioration of the sapphire's polished surface which causes premature breaking of the sapphire high pressure anvil, and therefore significantly improves the reliability of the system at high pressure. Other modifications to the system, such as the microwave coupling method, the modulation coil, and selecting of a hydrostatic pressure fluid, are discussed. EPR measurements on $\mathrm{BaTiO}_{3}$ have been made at temperatures ranging from 233 to $353 \mathrm{~K}$ and pressures from 0 to $4.4 \mathrm{GPa}$. High quality signals can be realized in the entire pressure and temperature range. (C) 1997 American Institute of Physics. [S0034-6748(97)01610-9]
\end{abstract}

\section{INTRODUCTION}

The electron paramagnetic resonance technique (EPR) is valuable in studies of structures of solid state material because of its high sensitivity to microscopic local fields. One of the fruitful applications of this technique is the study of structural phase transitions in solids because of the large changes in the observed spectra. EPR can also be used to investigate changes in the order parameter as a function of temperature and pressure. At low temperatures, EPR is especially useful for studies of ferroelectrics where dielectric measurements of the hysteresis loop can be hindered because the surface charges become so immobile. From EPR, the orientation and relative number of domains can be measured since the paramagnetic impurities are statistically distributed among them. The local point symmetry can also be determined.

In our laboratory, we are interested in studies of the microscopic mechanisms of phase transitions and critical phenomena, especially those transitions driven by a "soft mode." Since high pressure is an effective way to change the interatomic distance, and thus the mode frequencies in a solid, EPR studies with varying pressure and temperature are very useful in understanding the mechanism of phase transitions and critical phenomena in solids. EPR measurements at moderate hydrostatic pressures ${ }^{1,2}$ and at very high quasihydrostatic pressures have been reported. ${ }^{3,4}$ Data taken under quasihydrostatic conditions are always questionable due to unknown effects of nonhydrostatic stresses.

A hydrostatic high pressure EPR system was developed earlier in this laboratory. ${ }^{5}$ The microwave resonance cavity is made of a single crystal sapphire cylinder, which doubles as an anvil in a Bridgman-anvil metal-gasket pressure system.

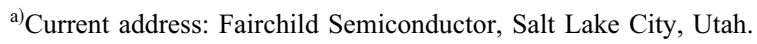

Being optically transparent, the sapphire crystal transmits the laser beam for pressure measurements using ruby fluorescence from a small crystal of ruby placed next to the sample in the hydrostatic fluid. The crystallographic $c$ axis of the sapphire anvil is along the cylinder. A compressive radial stress is added to the sapphire by a set of binding rings. To make a good conductive surface for the microwave cavity in the original system, the sapphire was coated with a piece of thin silver compound transfer tape, followed by baking at $850^{\circ} \mathrm{C}$, and cooling slowly. In order to get good microwave coupling, a micrometer was used to adjust the relative position of the microwave probe on the cavity. The pressure cell itself was built in an Eulerian cradle to rotate about a vertical and a horizontal axis with respect to the mount. Therefore this system was able to make EPR measurements with the sample and the cavity oriented in any direction with respect to the magnetic field. An eight-ton force-capacity hydraulic ram was incorporated directly within the cell. The high pressure was created in the fluid when the enclosing metal gasket surrounding the sample and ruby was compressed between the tapered opposing anvil and the sapphire cavity. This technique is similar to that commonly used in the gasketed diamond-anvil pressure cell. ${ }^{6}$ Tapered anvils with flat faces between 2 and $4 \mathrm{~mm}$ in diameter were used depending on the maximum pressure desired. The initial dimensions of the sample chamber formed in the gasket ranged from 0.8 to 1.5 $\mathrm{mm}$ in diameter and 0.3 to $0.6 \mathrm{~mm}$ in thickness. The smaller anvils are required to obtain higher pressures. By using the ruby-fluorescence pressure measuring technique, continuous pressure measurements could be easily made. EPR measurement at pressures as high as $6.0 \mathrm{GPa}$ have been reported using this system. ${ }^{5}$

Two of the major limitations of the system are (a) it cannot be cooled and controlled at temperatures lower than room temperature; (b) pressures above $2.5 \mathrm{GPa}$ cannot be 
reliably obtained due to the development of cracks on the sapphire cavity. Sapphire cavity breakage was related to the method used to silver coat the cavities. The previously used commercial silver transfer tape became unavailable and any substitution proved to be unsatisfactory.

Some major modifications to the original system have been made. One of the main improvements is the development of the low temperature capability. To obtain thermal isolation, the high pressure EPR cell is placed inside a vacuum chamber between the magnet pole faces. The cell is then cooled by a closed cycle refrigerator. Another improvement on the system is a new method of forming a conductive surface to define the microwave cavity. This method successfully avoids the deterioration by etching of the sapphire's polished surface caused by the previously used silver coating technique, which results in premature breaking of the sapphire. This approach significantly improves the reliability of the system at high pressure. These as well as other modifications to the system are discussed in detail in this article.

Recently, in this laboratory, we have been investigating the tetragonal-cubic phase transition in $\mathrm{BaTiO}_{3}$ using EPR. This transition is first-order at atmospheric pressure but at high enough pressure it becomes second-order. The pressuretemperature point where this change takes place is called a tricritical point. The nature of the transition near a tricritical point is of great interest. According to the dielectric and polarization measurements of Decker and Zhao, ${ }^{7}$ the tricritical point is near $3.5 \mathrm{GPa}$ and $233 \mathrm{~K}$. The new system enables us to do EPR investigations of $\mathrm{BaTiO}_{3}$ at pressures and temperatures in the vicinity of this tricritical point. Some of the EPR spectra on $\mathrm{BaTiO}_{3}$ are given to illustrate the use of the apparatus, but detailed results and discussion of $\mathrm{BaTiO}_{3}$ will be published separately.

\section{MODIFICATIONS AND IMPROVEMENTS OF THE APPARATUS AND TECHNIQUE}

In this article, we concentrate on the improvements recently made on the system. Readers who need more detailed information are referred to the original publication of Barnett et $a l^{5}$

In the present system, an $X$-band IBM Electron Spin Spectrometer ER 200D SRC, described in detail in IBM Instruments Inc. Publication No. GC22-9177, is used. The magnetic field is supplied by a Model L12-A Spectromagnetic Industries electromagnet with $30 \mathrm{~cm}$ pole faces and a $10 \mathrm{~cm}$ gap. Due to the dimensions of the pressure cell and the vacuum chamber, the $10 \mathrm{~cm}$ gap is necessary. The magnetic field power is supplied by a Varian V-FR 2100 Fieldial system. An IBM ER031 Field Controller connected with a semiconductor hall probe, placed on one of the magnet poles, is used for real-time measuring and controlling of the magnetic field. A Varian F-8 Nuclear Fluxmeter in combination with a Hewlett-Packard 5255-A Frequency Counter is used to calibrate the magnetic field at the sample position. An IBM Microwave Bridge ER $041 \mathrm{MR}$ is used to provide the microwave power and detect the absorption. The HewlettPackard 5255-A Frequency Counter is also used to give a precise measurement of the microwave frequency from the

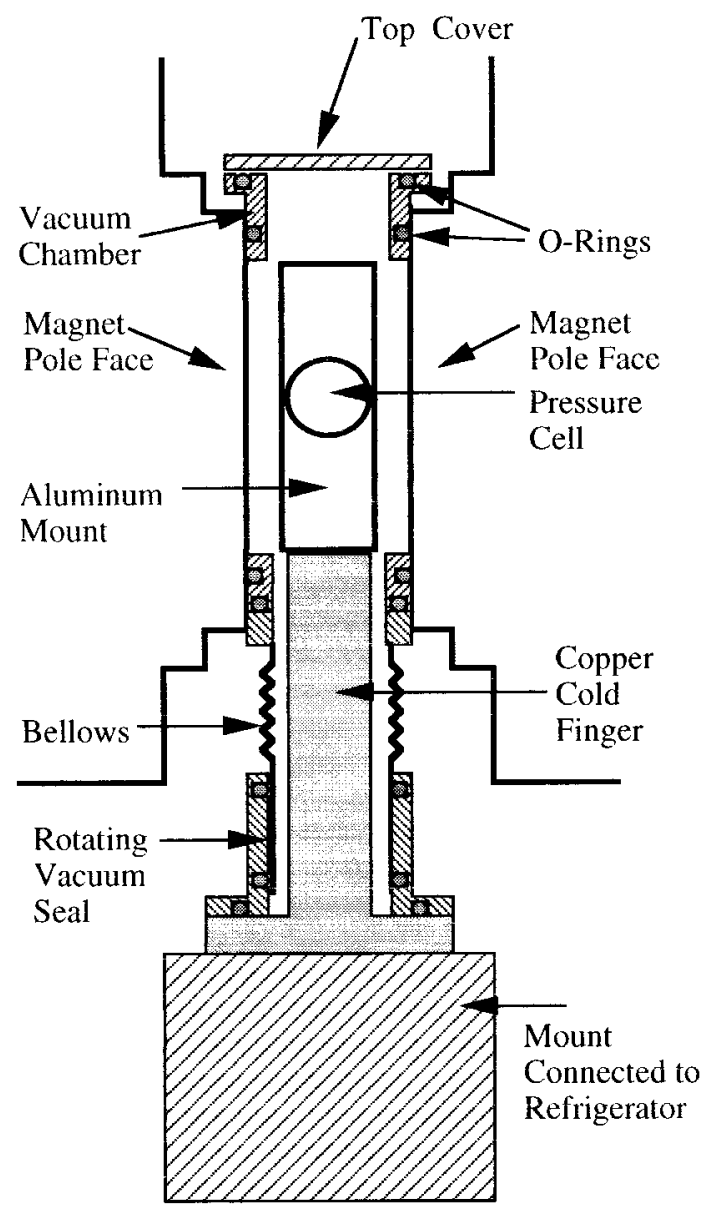

FIG. 1. The vacuum chamber containing the pressure cell.

microwave bridge. Some major improvements are discussed as follows.

\section{A. Low temperature capability}

To allow the cooling of the pressure cell, a vacuum chamber is constructed between the two pole faces of the electromagnet. An Air Product Displex refrigerator and temperature controller are used to cool the pressure cell. The configuration of the vacuum chamber is illustrated in Fig. 1.

The vacuum chamber is attached to and rotates with the electromagnet while the pressure cell and the displex refrigerator are stationary. The vacuum chamber is connected to an oil-diffusion pump and the pressure is kept at about $10^{-5}$ Torr during the measurements. With the temperature control unit, the sample can be cooled to temperatures between 45 to $60 \mathrm{~K}$, depending upon the energy input to the modulation coil, or heated to about $473 \mathrm{~K}$. Notice that a bellows is used to separate the rotating part and the fixed base. The center axis of the large rotating magnet may not be exactly the same as that of the fixed base, and the bellows gives a little flexibility for rotation.

A thermocouple is placed on the lower face of the refrigerator cold finger (Fig. 1) and used by the controller to control the temperature. Another thermocouple is located in a hole drilled in the pressure cell mount made of berylliumcopper. Since this thermocouple is very close to the sample and beryllium-copper is a good thermal conductor, the tem- 


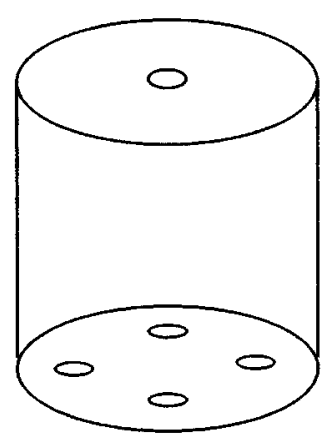

(a) Openings on the cavity

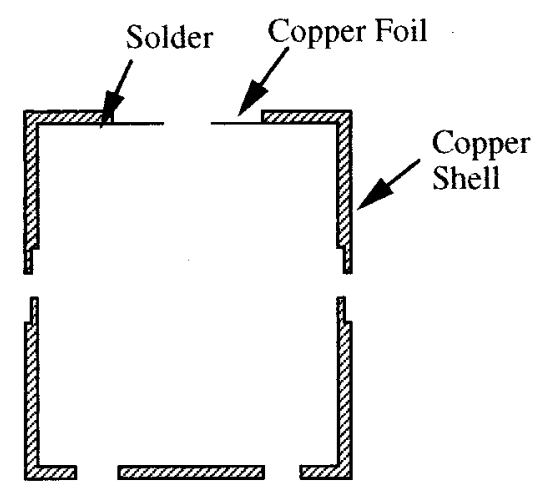

(b) Copper shell of the cavity

FIG. 2. The microwave resonance cavity.

perature measured by this thermocouple is considered to be the temperature of the sample. The temperatures at these two thermocouples are carefully measured and recorded allowing one to control the sample at the desired temperature by adjusting the controller. Given enough time for the system to reach equilibrium, the temperature at the sample can be controlled to a precision of $0.1 \mathrm{~K}$.

\section{B. High pressure microwave cavity}

The resonance cavity is made of a single crystal sapphire cylinder, made by Crystal System, which doubles as an anvil in a Bridgman-anvil metal-gasket pressure system. The dimensions (1.18 cm diam and $1.27 \mathrm{~cm}$ length) are selected to be consistent with the wave length of the klystron radiation within the dielectric. A thin copper shell with the same inner dimensions as the sapphire is machined and the sapphire is put in the copper shell to make a resonance cavity, as shown in Fig. 2. The thickness of the shell must be much greater than the skin depth of the microwave field $(f=9.3 \mathrm{kMHz})$ but much less than that of the modulation field $(f=100$ $\mathrm{kHz}$ ). The wall thickness of the shells used in our experiments is about $0.2 \mathrm{~mm}$. This cavity is excited in the $\mathrm{TM}_{110}$ cylindrical cavity mode. At the center of one of the ends of the cavity, a small hole (approximately $1.5 \mathrm{~mm}$ in diameter) is made in the thin copper shell and the experimental sample is placed in this area. Four holes (2 $\mathrm{mm}$ in diameter) are made in the shell at the opposite end of the cavity. Among these four, two opposite ones are used for optical access to measure the pressure and either one of the other two is used for the microwave coupling. Figure 2 shows these openings on the copper shell. The microwave coupling is capacitive in nature and is accomplished by a high-impedance coaxial input cable with an enlarged central conductor at its end. It is positioned at the opening where there is to be a high electric field for the specific mode desired. The coaxial cable is about $15 \mathrm{~cm}$ long and is connected directly to a rectangular wave guide associated with the IBM microwave detection system. The coaxial cable gives the flexibility needed to rotate the magnet since the vacuum chamber and thus the wave guide connected to it rotate with the magnet while the pressure cell remains fixed.

In the original system, there was a micrometer adjuster, used to adjust the relative position of the microwave coupling probe on the cavity in order to obtain optimal coupling. ${ }^{5}$ With this method, any slight movement of the coaxial cable influenced the coupling. In the new system, the coupling probe is tightly secured in contact with the cavity, and a mutual impedance bridge is added on the microwave guide. This approach provides a much more reliable coupling not affected by the movement of the cable. This is very important for the low temperature system because the coupling adjustment cannot be made outside of the vacuum chamber which rotates with respect to the pressure cell when the direction of the magnetic field is changed.

The newly designed modulation coil is composed of three Helmholz coils set in orthogonal directions. The new coil allows changing the direction of the modulation field by moving a wiring jumper, which controls the magnitude and the direction of the currents in each Helmholz coil. One can thus select an optimal combination for the direction of the modulation field with respect to the dc magnetic field. The modulation coil is fixed on the pressure cell mount. When the big magnet rotates, the modulation coil does not. This new feature of the modulation coil allows us to adjust the modulation field after rotating the magnet to maintain strong EPR signals.

Not only does the single crystal sapphire serve as the microwave cavity and a high pressure anvil but being optically transparent it transmits the laser beam for pressure measurement using ruby fluorescence from a small crystal of ruby placed next to the sample in the hydrostatic fluid. The opposing anvil is made of chromium carbide instead of polycrystalline $\mathrm{Al}_{2} \mathrm{O}_{3}$ used in the original system. Chromium carbide is paramagnetic in the temperature range used. Chromium carbide has a greater tensile strength than polycrystalline $\mathrm{Al}_{2} \mathrm{O}_{3}$, so the anvil can be used without a binding ring, and therefore leaves more space for the modulation coil. The gasket between the two anvils is made of inconel which is also nonmagnetic in the temperature range. The thickness of the gasket is approximately $0.3 \mathrm{~mm}$. A hole with a diameter of $1.1 \mathrm{~mm}$ is drilled through the center of the gasket into which is placed the crystal sample and the ruby fragment.

Although sapphire crystals have very high compressive strength, their tensile strength is low. It is important to have a compressive stress in the radial direction before applying pressure along the axial direction. This is accomplished by 


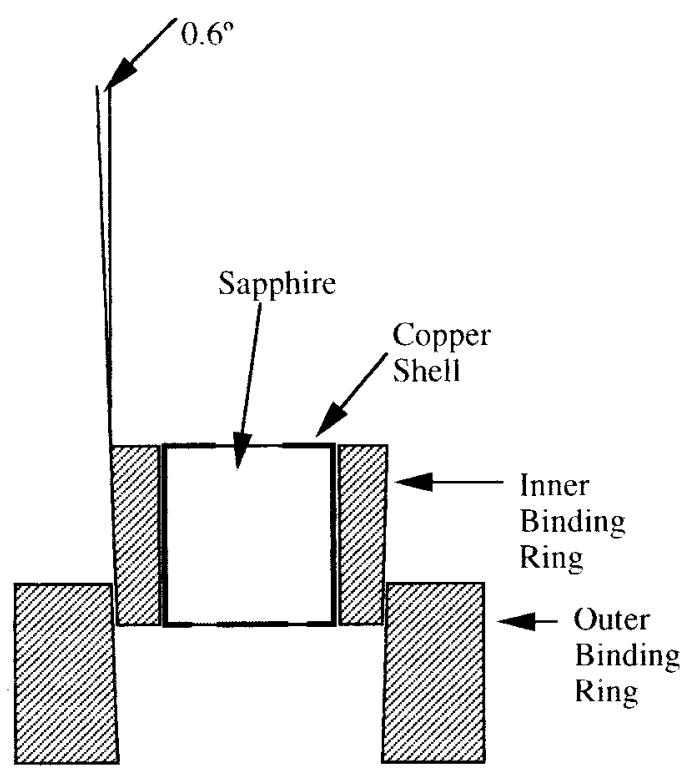

FIG. 3. The binding rings and the sapphire.

two binding rings as shown in Fig. 3. The rings are made of titanium since titanium is nonmagnetic and has a thermal expansion coefficient close to that of the sapphire; so that the radial stress is not temperature dependent. The inner and outer rings are tapered with a half-cone angle of $0.6^{\circ}$, producing an overlap of 0.06 to $0.08 \mathrm{~mm}$ in the diameters. When the inner ring is pressed into the outer ring, a radial compressive stress is added to the sapphire. The stress distribution inside the sapphire was calculated by numerical modeling. The stronger the radial compressive stress supplied by the binding rings the better the sapphire can support the axial pressure, up to the limit of the compressive strength of the sapphire.

A microwave cavity must have a good conductive surface. In the original system, the sapphire was coated with silver by using a piece of thin silver compound transfer tape followed by baking at $850{ }^{\circ} \mathrm{C}$, and cooling slowly. This made a high $Q$ cavity. The major problem was that sapphire anvils treated in this manner tended to crack upon pressure release when pressures above $2.5 \mathrm{GPa}$ were used. When the low temperature capability was developed, the silver compound transfer tape was no longer available. A silver conductive composition paste was used to paint the sapphire surface, and then the sapphire was baked at $850^{\circ} \mathrm{C}$. With sapphire anvils prepared in this manner, cracks developed even earlier upon application of pressure, and the highest pressure achieved was about $2.0 \mathrm{GPa}$. It is proposed that when the sapphire is heated the bonding agent in the silver compound attacks the surface producing defects which develop into cracks when the sapphire is under stress. The surfaces before and after silver coating were analyzed by a Wyco Surface Profiler and the results are shown in Fig. 4. It is obvious that the roughness of the surface increased significantly in the silver coating process. For this reason, we designed the copper cans as a conductor to define the cavity as shown in Fig. 2. This copper shell preserved the high polish
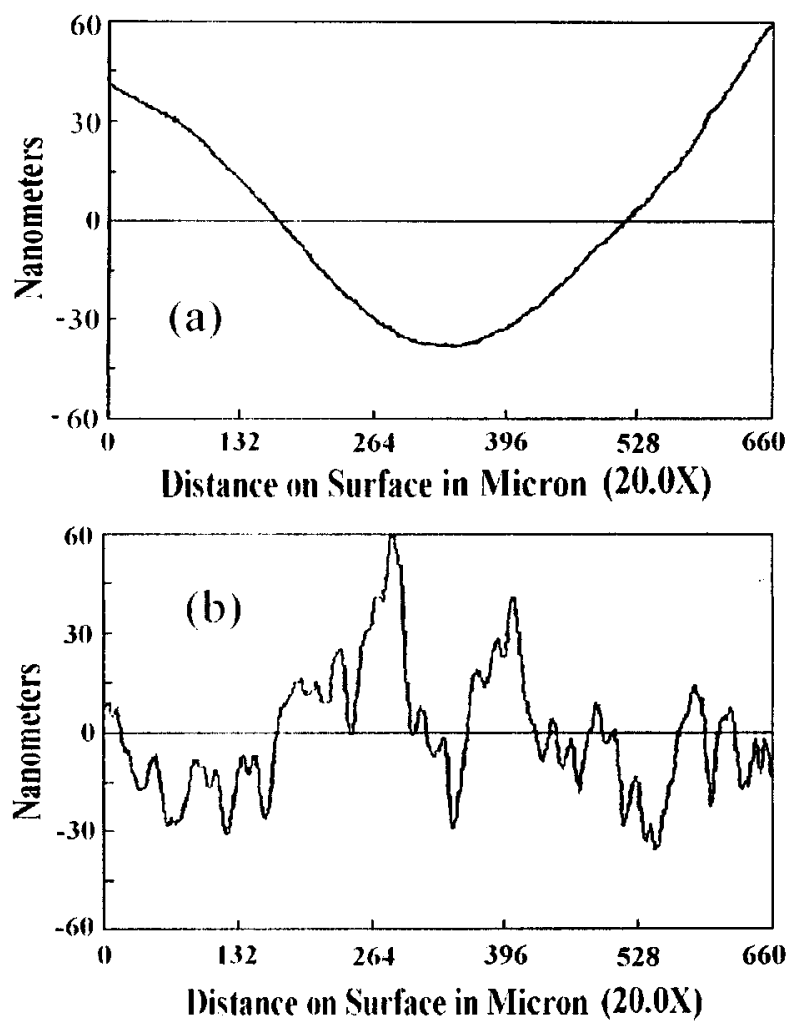

FIG. 4. The surface of the sapphire analyzed by the WYKO surface profiler: (a) original surface, (b) the surface after being painted with a silver compound and baked at $850^{\circ} \mathrm{C}$.

on the sapphire surface, and essentially eliminated the cracking at high pressure.

Petroleum ether is used as the hydrostatic pressure fluid surrounding the sample. A methyl/ethyl alcohol mixture is commonly used as a hydrostatic pressure fluid at low temperature and high pressure. When using a resonance cavity, where the conductive surface is not intimately adhered to the sapphire, the pressure transmitting liquid flows into the space between the sapphire and the conductor, and this severely deteriorates the $Q$ of the cavity when using the methyl/ethyl liquid. Since petroleum ether does not absorb the $X$-band radiation, no $Q$ deterioration is observed. How the $Q$ is affected by a fluid is related to the dielectric property of the molecules of the fluid and impurities absorbed by the fluid.

Both petroleum ether and methyl/ethyl alcohol evaporate quickly. Thus care must be taken during the sample loading of the pressure system to insure that there is still sufficient fluid to fill the cell when sealed under pressure. Figure 5 shows the configuration just before pressure is applied. The sample and the ruby are positioned on the sapphire cavity at the center of the opening in the copper shell using a low power microscope, and are secured with clear Krylon spray. The gasket is put on top of the copper shell with the sample and the ruby seated inside its central hole. To hold the pressure, the central part of the high pressure end of the copper shell has to be very thin (see Fig. 2). Because of its extreme thinness, this part cannot be machined as is the rest of the shell. A thin copper foil is soldered to this face of the copper can. Being very thin and not secured to the sapphire, it may 


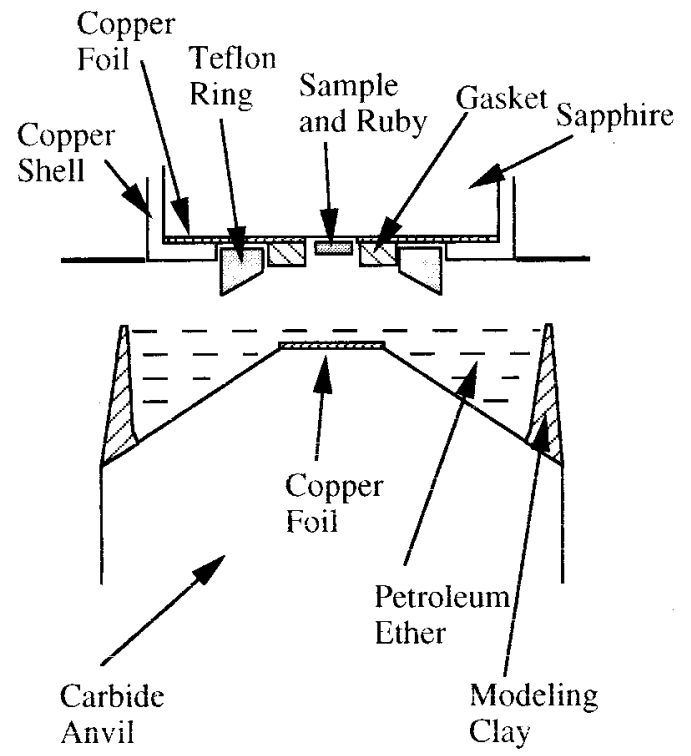

FIG. 5. The system just prior to applying pressure.

vibrate in the modulation magnetic field and produce Eddy currents which absorb energy and cause a serious baseline drift in the EPR magnetic field signal. To avoid the free movement of the copper foil, a Teflon ring is put outside the gasket (Fig. 5). The Teflon ring is machined to a shape such that when the system is under pressure it will hold the copper foil tightly against the sapphire. Both the Teflon ring and the gasket are secured to the copper shell by Duco cement.

\section{Ruby-fluorescence pressure measurement}

The basic principle of the ruby-fluorescence pressuremeasuring system is based on the fact that when a piece of ruby is illuminated with high intensity light, the wavelengths of the $R_{1}$ line in the fluorescence spectrum has a nearly linear pressure dependence and has been well calibrated. ${ }^{8}$ The setups for pressure measurement in the present system are very similar to the one described by Barnett et al. ${ }^{5}$

In the original system, the incident laser light and the ruby-fluorescence light passed through the same window hole on the sapphire cavity. In the new system, two opposite holes, as shown in Fig. 2, are used for pressure measurement, one for the incident laser beam and the other for the ruby fluorescence. This makes the precise optical adjustment easier.

The laser used to excite the ruby fluorescence is a Spectra-Physics Model 161 Argon-15 milliwatt laser with a wavelength of $488 \mathrm{~nm}$. When the monochromator sweeps, the intensity of the fluorescence at different wavelengths is converted to a digital signal and processed by a microcomputer. The computer compares the position and shape of the fluorescence peaks to that of the standard reference taken at atmosphere pressure and calculates the pressure according to the calibration taking into account the effect of temperature. This pressure-measuring system provides reproducible measurements with a precision of approximately $50 \mathrm{MPa}$. It is important that the laser beam direction and position be adjustable with reasonable high precision to facilitate directing

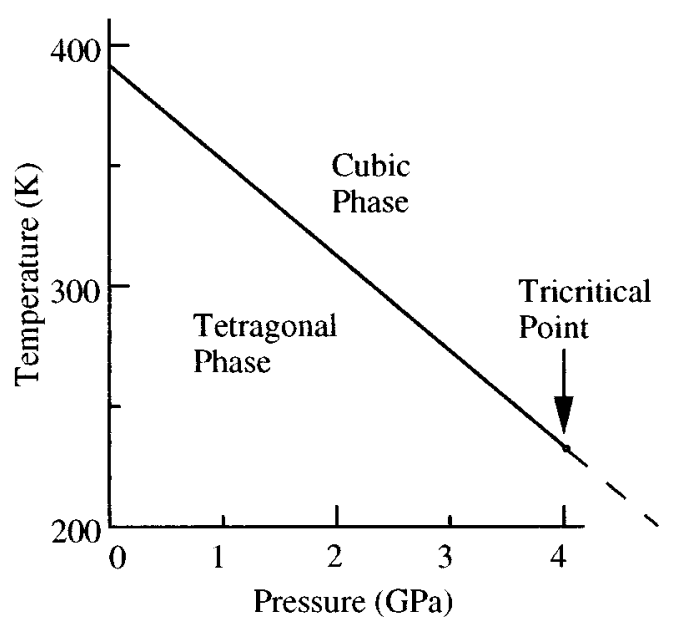

FIG. 6. The phase line of the ferroelectric transition of $\mathrm{BaTiO}_{3}$.

the beam onto the ruby fragment within the pressure cell since the cell cannot be moved easily after being positioned in the vacuum chamber.

\section{EPR MEASUREMENTS ON $\mathrm{BaTiO}_{3}$}

To illustrate the capabilities of the improved high pressure EPR system, we report here some EPR data on $\mathrm{BaTiO}_{3}$. The phase line of the ferroelectric transition is shown in Fig. 6 . The purpose of the measurements on $\mathrm{BaTiO}_{3}$ is to investigate changes in the EPR signals near the tetragonal-cubic phase line, especially in the neighborhood of the tricritical point where the transition changes from first-order to secondorder. The single-crystal $\mathrm{BaTiO}_{3}$ doped with 0.03 wt. \% $\mathrm{Fe}_{2} \mathrm{O}_{3}$ was grown by Albers with the top-seeded solution technique. ${ }^{9}$ This crystal has sharper EPR lines than the "butterfly" crystals and other crystals formerly used for EPR measurements in this lab. ${ }^{10}$ The sample is polished to a 0.1 $\mathrm{mm}$-thick plate and then cut to a round disk of $0.6 \mathrm{~mm}$ diam. The disk is oriented such that one of the $a$ axes is perpendicular to the plate while the other $a$ axis and the $c$ axis lie in the plane of the plate.

The $\mathrm{Fe}^{3+}$ in $\mathrm{BaTiO}_{3}$ has a spin of 5/2. The five dominant resonance lines are produced by the transitions: $+5 / 2$ $\rightarrow+3 / 2,+3 / 2 \rightarrow+1 / 2,+1 / 2 \rightarrow-1 / 2,-1 / 2 \rightarrow-3 / 2$, and $-3 /$ $2 \rightarrow-5 / 2$. In most situations only four or sometimes three of these lines are observed because the broadness of the lines leaves insufficient signal or the resonance is out of range of the available magnetic field. EPR data along the $c$ and $a$ axes will give us enough information for our analysis. Therefore only EPR measurements along these two directions are taken under pressure. Figures 7(a)-7(c) show three lines measured along the $c$ axis at $2.5 \mathrm{GPa}$ and $243 \mathrm{~K}$. The $+1 / 2 \rightarrow-1 / 2$ line has a differential signal peak-to-peak width of about $11 \mathrm{G}$ and the other two lines are above $45 \mathrm{G}$ wide. The EPR signals are sufficient for accurate analysis of the resonant fields. Some background slope is evident in Fig. 7(a). Similar quality signals are observed along the $a$ axis. EPR measurements are made at temperatures ranging between 233 and $353 \mathrm{~K}$ and pressures from 0 to $4.4 \mathrm{GPa}$. At $4.4 \mathrm{GPa}$, the sapphire is not broken and can be reused, however the sample will be 

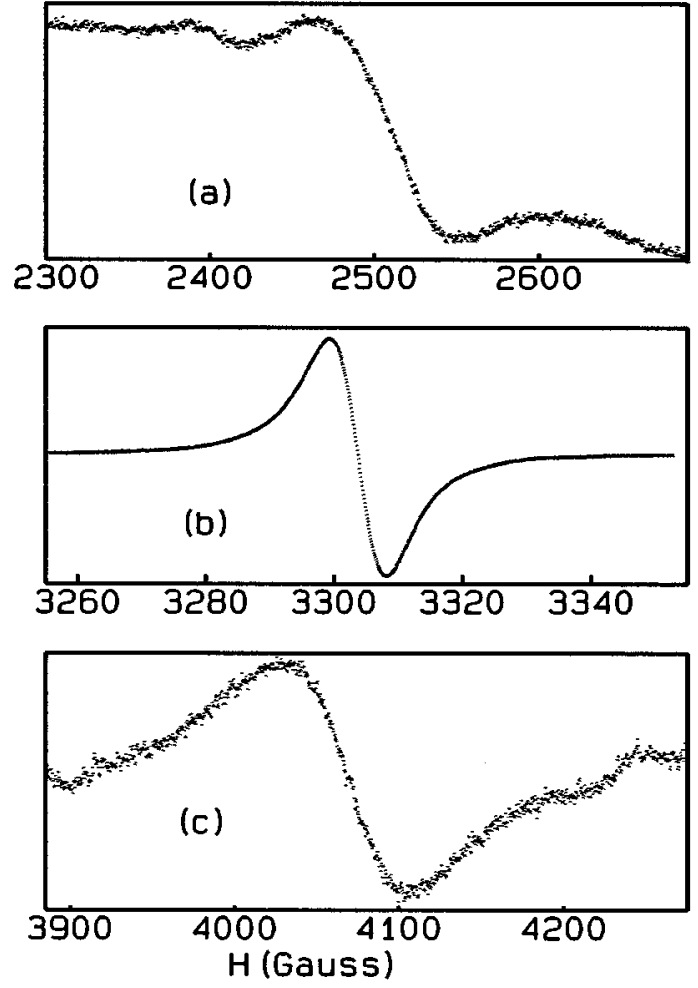

FIG. 7. EPR signals of $\mathrm{BaTiO}_{3}$ with the magnetic field along the $c$ axis at $2.5 \mathrm{GPa}$ and $243 \mathrm{~K}$.

smashed at the highest pressures if the gasket has been compressed such that it is thinner than the crystal. One must use thinner samples to go to higher pressures.

\section{DISCUSSION}

We have presented an improved EPR system capable of measurements in a hydrostatic environment to the same pressure range as the original hydrostatic system developed in this laboratory, but the system presented here is more reliable at high pressure and also has the capability of making measurements at low temperatures. Thus this system extends the thermodynamic region over which meaningful EPR measurements can be made. The EPR measurements on $\mathrm{BaTiO}_{3}$ illustrate that the data is very good even with small samples dictated by the size of the pressure cell.
In the original system, ${ }^{5}$ at pressures above $2.5 \mathrm{GPa}$, the sapphire cavity often developed cracks upon pressure release, and the cavity could not be used again. We employ a new method that preserves the original polish on the sapphire surface and thus improves the pressure supporting ability of the sapphire. We have used a sapphire anvil repeatedly at pressures above 4.0 GPa without breaking.

Because of the capability to make continuous internal pressure measurements, this system is very suitable for measurements of variations of the spin Hamiltonian parameters as well as measurements of linewidths at different pressures and temperature since the parameters are continuous and can be measured at any arbitrary number of points within the pressure range of interest. Measurements on $\mathrm{BaTiO}_{3}$ have yielded very useful information about the spin Hamiltonian parameters. Detailed results and discussion on measurements of $\mathrm{BaTiO}_{3}$ will be published separately.

The main limitation of the system is the small sample volume. The higher the desired pressure the smaller the sample must be. Signal strength is always important, especially when the temperature and pressure are close to a phase transition, where the EPR lines are broadened by the critical fluctuations.

\section{ACKNOWLEDGMENTS}

The authors would like to thank Wesley Lifferth who constructed the pressure cell, the Eulerian cradle and the copper cavity shell, and Dr. Brett Hess for performing the test of the system at low temperature.

${ }^{1}$ W. M. Walsh, Jr. and W. Bloembergen, Phys. Rev. 107, 904 (1957).

${ }^{2}$ J. Stankowski, A. Galezewski, M. Krupski, S. Waplak, and H. Gierszal, Rev. Sci. Instrum. 47, 128 (1976).

${ }^{3}$ L. Rimai, T. Deutsch, and B. D. Silverman, Phys. Rev. 133, A1123 (1964).

${ }^{4}$ H. M. Nelson, D. B. Larson, and J. H. Gardner, J. Chem. Phys. 47, 1994 (1967).

${ }^{5}$ J. D. Barnett, Som D. Tyagi, and H. M. Nelson, Rev. Sci. Instrum. 49, 348 (1978).

${ }^{6}$ C. Weir, S. Block, and G. Piermarini, J. Res. Natl. Bur. Stand. Sect. C 69, 275 (1965).

${ }^{7}$ D. L. Decker and Y. X. Zhao, Phys. Rev. B 39, 2432 (1989).

${ }^{8}$ G. J. Piermarini, S. Block, J. D. Barnett, and R. A. Forman, J. Appl. Phys. 46, 2774 (1975)

${ }^{9}$ K. A. Muller, W. Berlinger, and J. Albers, Phys. Rev. B 32, 5837 (1985).

${ }^{10}$ M. H. Ellwanger, M.S. thesis, Brigham Young University, 1984. 